\title{
La stratégie de l'Union européenne contre la criminalité organisée : entre lacunes et inquiétudes
}

The European Union strategy against organised crime: between deficiency and concern

\section{Amandine Scherrer, Antoine Mégie et Valsamis Mitsilegas}

\section{(2) OpenEdition}

\section{Journals}

Édition électronique

URL : https://journals.openedition.org/conflits/17442

DOI : $10.4000 /$ conflits. 17442

ISSN : $1777-5345$

Éditeur :

CECLS - Centre d'études sur les conflits - Liberté et sécurité, L'Harmattan

Édition imprimée

Date de publication : 1 septembre 2009

Pagination : 91-110

ISBN : 978-2-296-09110-8

ISSN : 1157-996X

\section{Référence électronique}

Amandine Scherrer, Antoine Mégie et Valsamis Mitsilegas, «La stratégie de l'Union européenne contre la criminalité organisée : entre lacunes et inquiétudes », Cultures \& Conflits [En ligne], 74 | été 2009, mis en ligne le 28 octobre 2010, consulté le 08 décembre 2022. URL : http://journals.openedition.org/ conflits/17442 ; DOI : https://doi.org/10.4000/conflits.17442

\section{(c) (†) $\ominus$

Creative Commons - Attribution - Pas d'Utilisation Commerciale - Pas de Modification 4.0 International - CC BY-NC-ND 4.0

https://creativecommons.org/licenses/by-nc-nd/4.0/ 


\title{
La stratégie de I'Union européenne contre la criminalité organisée : entre lacunes et inquiétudes
}

\author{
Amandine SCHERRER, Antoine MEGIE, Valsamis MITSILEGAS
}

Amandine Scherrer est docteur en science politique, actuellement chercheur invité à l'université de Manchester (Royaume-Uni). Ses recherches portent sur les processus d'élaboration des normes internationales contre la criminalité organisée, ainsi que sur leur circulation sur la scène internationale. Elle a récemment publié un ouvrage sur ces questions, intitulé G8 against Transnational Organized Crime (Ashgate Publishing, 2009), et a également contribué à un ouvrage collectif sur le crime organisé coordonné par Felia Allum (University of Bath), Francesca Longo (université de Catane) et Daniela Irrera (université de Catane) : Defining and Defying Organised Crime: Discourse, Perceptions and Reality, à paraître chez Routledge.

Antoine Mégie est docteur en science politique, actuellement ATER à l'université de Versailles St-Quentin/CARPO et coordinateur du projet ANR Polilexes (Paris I La Sorbonne). Ses recherches portent principalement sur la construction européenne en matière de justice pénale ainsi que sur la question du terrorisme et de l'antiterrorisme. Il a récemment publié un article sur ces questions, "La lutte antiterroriste et les libertés publiques : les ambigüités européennes ", dans la revue Questions internationales ( $n^{\circ} 34$, novembre 2008) et prépare actuellement un article pour la revne Droit et Société, "Vers la construction d'une expertise européenne en matière de coopération pénale ? Spécialisation et légitimation des professionnels de la coopération judiciaire».

Valsamis Mitsilegas est docteur en droit et enseigne à la Queen Mary University à Londres. Ses recherches et son expertise concernent tout d'abord le domaine des affaires criminelles au sein de l'UE. Il s'intéresse également au développement des réponses institutionnelles apportées contre la criminalité organisée, le blanchiment d'argent, et le terrorisme, aussi bien au niveau national qu'international. De 2001 à 2005, il a été conseiller juridique auprès du Comité pour l'UE de la chambre des Lords, et est ainsi régulièrement consulté par les Parlements, les organisations 
internationales et les ONG. Il est également expert auprès $d u$ Comité $d u$ Parlement européen pour les libertés civiles, justice et affaires intérieures (LIBE). Il a récemment publié un ouvrage sur ses questions : EU Criminal Law (Hart Publishing, 2009).

$\mathrm{D}$ epuis la fin des années 1980, la criminalité transnationale organisée (CTO) est un élément majeur des agendas des institutions internationales ${ }^{1}$. La mobilisation de la communauté internationale a donné lieu à l'élaboration d'un nombre impressionnant de réponses institutionnelles à ce qui a été décrit comme un défi d'envergure mondiale ${ }^{2}$. L’Union européenne, au sein de son agenda Justice-Affaires intérieures (JAI), n'a pas fait exception dans cette mobilisation, et a constamment inclus cet aspect dans ses préoccupations depuis le milieu des années 1990.

Cependant, et malgré une mobilisation internationale toujours croissante, les différentes expériences et tentatives pour harmoniser la lutte internationale contre la criminalité organisée se sont toutes heurtées à la difficulté de définir la CTO au niveau global. Cet article tentera de montrer que définir et criminaliser la criminalité organisée est une tâche complexe, étant donné la variété des activités des groupes criminels et l'hétérogénéité des degrés d'organisation de tels groupes ${ }^{3}$. Ces difficultés sont centrales dans la compréhension de l'état actuel de la mobilisation européenne contre la CTO. En effet, alors que les définitions des concepts restent vagues et imprécises, des mesures législatives sont adoptées, notamment dans le domaine pénal, ainsi que des instruments et des mécanismes opérationnels. Les plus récentes tentatives de définition de la criminalité organisée, notamment au sein de la décision-cadre du Conseil de 2008 , illustrent à nouveau cet aspect problématique et sensible de l'internationalisation de la lutte contre la criminalité organisée.

De plus, une étude approfondie de ce phénomène d'internationalisation, dans lequel l'UE a joué un rôle important, montre des glissements ainsi que des lacunes importantes. De nombreuses analyses ont par exemple montré que, selon les environnements politiques et les intérêts professionnels, la lutte internationale contre la criminalité organisée a constamment évolué, se focalisant alternativement sur le trafic de stupéfiants, le blanchiment d'argent, ou encore le financement du terrorisme 5 . De la même manière, d'autres éléments habituellement englobés dans le concept très vague de CTO, comme la corruption, le crime d'entreprise, ou la criminalité en col blanc, sont demeurés secondaires dans l'ordre des priorités des agendas internationaux et euro-

1. Cet article reprend en grande partie une note rédigée pour le Parlement européen en janvier 2009. Nous remercions M. Davoli d'avoir accepté cette publication dans la revue Cultures $\mathcal{E}$ Conflits.

2. Beare M., Critical Reflections on Transnational Crime, Money Laundering, and Corruption, Toronto, University of Toronto Press, 2003 ; Edwards A., Gill P., Transnational Organised Crime. Perspectives on global security, London, Routledge, 2003 ; Scherrer A., G8 against Transnational Organized Crime, Aldershot, Ashgate, 2009. 
péens, tout du moins en comparaison avec le blanchiment d'argent ou le financement du terrorisme.

Afin de rendre compte de ces lacunes et de ces inquiétudes, et de mieux comprendre ainsi l'état actuel de la stratégie de lutte de l'UE contre la criminalité organisée, cet article examinera dans un premier temps le cadre juridique de l'UE dans ce domaine. Les aspects opérationnels de la stratégie de l'UE feront l'objet d'une seconde partie, qui sera dédiée à la coopération judiciaire et policière et au rôle des agences européennes comme Europol et Eurojust.

\section{Le cadre juridique de l'Union européenne contre la criminalité organisée}

Cette partie a pour objet la présentation des divers instruments législatifs adoptés dans le cadre de l'UE afin de lutter contre la criminalité organisée, et ce dans trois domaines de l'action de l'Union : les mesures qui criminalisent la participation à une organisation criminelle ; les mesures de lutte contre le blanchiment d'argent (mesures répressives et préventives); les mesures de lutte contre la corruption.

\section{L'infraction de "criminalité organisée ": des difficultés de définition persistantes}

La lutte contre la criminalité organisée a constitué un élément central de l'agenda JAI de l'UE, comme en témoignent les deux plans d'action pour lutter contre la criminalité organisée de 1997 et de 2000, ainsi que les conclusions de Tampere et le programme de La Haye de 2004. La criminalisation de la participation à une organisation criminelle est un élément central dans ce contexte. La définition des activités relevant du crime organisé, et la reconnaissance de leur caractère criminel, constituent le cœur des politiques pénales contre ce phénomène. Cet aspect est une des tâches les plus primordiales dans le souci de définir et de clarifier le mandat des organes de coopération policière et judiciaire de l'UE que sont Europol et Eurojust, tous deux ayant pour principale mission la lutte contre la criminalité organisée. Cependant, la cri-

3. Beare M., Naylor R.T., Major Issues Relating to Organized Crime (Report for the Law Commission of Canada), 1999 ; Mitsilegas V., "From National to Global, from Empirical to Legal: The Ambivalent Concept of Transnational Organized Crime”, in Beare, op. cit., 2003 ; Sheptycki J., “Against Transnational Organized Crime”, in Beare, op. cit., 2003.

4. Décision-cadre 2008/841/JAI du Conseil du 11 novembre 2008 relative à la lutte contre la criminalité organisée, JO L 300, 11 novembre 2008.

5. Naylor R.T., Wages of Crime. Black Markets, Illegal Finance and the Underworld Economy, Ithaca, Cornell University press, 2002 ; Mitsilegas V., "Countering the Chameleon Threat of Dirty Money. 'Hard' and 'Soft' Law in the Emergence of a Global Regime Against Money Laundering and Terrorist Finance", in Edwards, Gill, op. cit., 2003 ; Beare M., "Fear-Based Security: The Political Economy of Threat”, Papier présenté à l'International Terrorism and Money Laundering Transnational Institute (TNI) Amsterdam, 2005 ; Naylor, R.T., Satanic Purses: Money, Myth, and Misinformation in the War on Terror, Montréal, McGill University Press, 2006 ; Scherrer A., op. cit. 
minalisation de la participation à une organisation criminelle est une entreprise complexe d'un point de vue juridique. Elle est en effet difficile à inscrire dans une norme assurant une certitude et une précision légales suffisantes face aux activités multiples des criminels organisés. Des questions épineuses se posent particulièrement en ce qui concerne la définition juridique des groupes criminels organisés, notamment concernant le degré d'organisation requis, la structure (ou non) de ces groupes et le nombre de personnes impliquées. D'autres sujets difficiles incluent les requis mens rea, c'est-à-dire le degré de connaissance ou d'intention requis pour qu'une personne puisse être poursuivie pour participation à des activités de crime organisé, mais également le degré de participation effective requis pour qu'il puisse y avoir criminalisation. Le problème majeur, comme pour l'infraction terroriste, est donc qu'il y a un risque de criminalisation du simple soutien des objectifs d'un groupe sans qu'il n'y ait d'implication dans un acte criminel. A cette complexité s'ajoutent les différences entre Etats membres de l'UE dans le traitement pénal du crime organisé. Nombre d'Etats membres n'ont d'ailleurs pas inclus d'infractions de criminalité organisée spécifiques dans leur droit pénal.

L'Union européenne a tenté de surmonter ces difficultés en 1998 par une action commune du troisième pilier dans le but de faire de la participation à une organisation criminelle dans l'UE une infraction criminelle. Cette action commune ${ }^{6}$ constitue une tentative ambitieuse de définir légalement les groupes criminels organisés tout en tenant compte des perceptions policières. Elle criminalise la participation active à une telle organisation et, alternativement, la conspiration en vue de commettre une des infractions mentionnées (article 2). Le recours à ces deux approches alternatives très différentes de la criminalisation est frappant pour un instrument qui tente d'harmoniser le droit pénal. Mais il peut être expliqué par la nécessité d'obtenir un compromis - un accord unanime au Conseil - malgré des approches juridiques nationales du crime organisé très différentes (avec l'alternative de la conspiration qui satisfait particulièrement aux exigences de la tradition britannique).

L'adoption de la Convention onusienne de Palerme de 2000 au niveau européen a conduit à la modification de l'action commune de l'UE de 1998, afin de mettre en conformité le cadre législatif européen avec la Convention. Ces modifications sont apportées par la récente adoption de la décision-cadre relative à la lutte contre la criminalité organisée 7 . La décision-cadre de 2008 relative à la lutte contre la criminalité organisée suit les provisions de la convention de Palerme et remplace l'action commune de l'UE de $1998^{8}$. Le texte montre ainsi une nouvelle tentative de définir la criminalité organisée. Cependant, les termes utilisés demeurent inévitablement imprécis (en particu-

6 . (98/733/JAI, OJ L351/1). Pour une analyse détaillée de l'action commune et des sujets traités dans le paragraphe 3, voir Mitsilegas V., "Defining Organised Crime in the European Union: The Limits of European Criminal Law in an Area of Freedom, Security and Justice", European Law Review, vol.26, 2001, pp. 565-581. 
lier en ce qui concerne une association «structurée » et le degré de "structure » requis) et s'accommodent mal de la nécessité de la clarté de la règle de droit. De plus, le Conseil a maintenu le modèle de l'action commune et de la convention de Palerme en donnant aux Etats membres le choix entre criminaliser la participation à une organisation criminelle ou la conspiration et, dans une nouvelle tentative d'éviter les affrontements entre traditions juridiques, s'est abstenu de criminaliser la direction d'un groupe criminel organisé 9 . La Commission s'est d'ailleurs opposée à ces deux éléments ${ }^{10}$.

La décision-cadre inclut désormais des mesures détaillées relatives aux peines encourues (minimum de 2 ans, maximum 5 ans, et la circonstance aggravante de l'infraction commise dans le cadre d'une organisation criminelle ${ }^{11}$ ), ainsi que des mesures sur la responsabilité des personnes morales 12 et sur les champs de compétence des juridictions ${ }^{13}$. Pour ce qui est des mesures concernant les circonstances aggravantes, certains des éléments précisés pourraient avoir un impact substantiel sur les systèmes juridiques nationaux (particulièrement à la lumière des décisions de la CEJ dans le cas Pupino), notamment ceux établissant des circonstances particulières ${ }^{14}$ et ceux établissant l'absence d'obligation de déclaration ou d'accusation émanant de la victime dans la conduite des enquêtes et des poursuites ${ }^{15}$. La Commission pointe peut-être quelque chose d'important lorsqu'elle critique le fait de maintenir dans une mesure d'harmonisation deux options alternatives de criminalisation du crime organisé. En effet, cela ne contribue pas à la certitude juridique et conduit potentiellement à une conception très extensive de la criminalisation de la criminalité organisée à travers l'UE. Les deux infractions alternatives sont décrites en des termes très généraux - le concept d'organisation criminelle est très large et la conspiration ne suppose pas forcément l'implication effective dans une activité criminelle. Ce qui est plus inquiétant est que cela peut conduire à une diversité d'application considérable à un moment où le «crime organisé » représente une infraction pour laquelle la criminalité duale a été abolie par les instruments de reconnaissance mutuelle (incluant le mandat d'arrêt européen, le mandat européen d'obtention de preuves et les mesures sur le gel et la confiscation de biens). De surcroît, le caractère vague de l'infraction de criminalité organisée est de fait inclus dans le mandat

7. Décision-cadre du Conseil 2008/841/JAI, OJ L 300, 11 novembre 2008, p. 42.

8. Préambule, considérant 6.

9. Article 2.

10. La Commission, à laquelle la France et l'Allemagne se sont jointes, a produit une déclaration attachée au texte soulignant que la décision-cadre ne parvenait pas à instaurer un minimum d'harmonisation des actes de direction ou de participation à une organisation criminelle, permettant ainsi aux Etats membres de ne pas introduire le concept d'organisation criminelle et de continuer à appliquer la législation existante. Voir document du Conseil doc. 9067/06.

11. Article 3.

12. Articles 5 et 6 .

13. Article 7.

14. Article 4.

15 . Article 8 . 
d'Europol et d'Eurojust. Par conséquent, ces derniers pourraient être appelés à agir contre des comportements qui seraient considérés comme de la criminalité organisée dans un Etat membre mais pas dans un autre.

\section{Le blanchiment d'argent}

La lutte contre le blanchiment d'argent est très étroitement liée à la lutte contre la criminalité organisée, la nécessité de retirer aux criminels les profits de leurs crimes ayant été centrale et présentée comme impérative dans la mise en place de mesures contre la CTO. Au cours de ces dernières années, la communauté européenne a développé un régime complet et sophistiqué contre le blanchiment d'argent. L'adoption récente de la troisième directive parachève ces développements. L'élaboration de cette troisième directive ${ }^{16}$ se voyait justifiée par la nécessité de conformer le cadre législatif européen aux nouveaux standards adoptés par le Groupe d'action financière international (GAFI) ${ }^{17}$. L'influence de ce dernier apparaît clairement à de nombreuses occasions dans le texte. Cette directive remplace les précédentes et des modifications majeures apparaissent dès l'intitulé de l'instrument, qui inclut désormais non seulement le blanchiment d'argent mais aussi le « financement du terrorisme ». La mise en conformité avec les standards internationaux se retrouve explicitement dans le préambule du texte, qui fait mention de références spécifiques à la menace terroriste ${ }^{18}$, à la nécessité de tenir compte des travaux du GAFI ${ }^{19}$, ainsi qu'à la modification des mesures concernant l'identification des clients dans le secteur bancaire à la lumière des derniers développements internationaux dans ce domaine ${ }^{20}$. Les modifications majeures qu'apporte cette troisième directive incluent, tout d'abord, l'amendement de la définition du blanchiment d'argent afin de l'aligner sur la définition de l'« infraction grave » telle que présentée dans la décision-cadre de 2001 relative à la confiscation (étendant ainsi le champ de l'infraction) ${ }^{21}$. Cette directive introduit également l'interdiction, en plus du blanchiment d'argent, du financement du terrorisme. À cet égard, la définition $\mathrm{du}$ « financement du terrorisme » est similaire, mais pas identique, à celle adoptée dans la Convention des Nations unies de 1999. Elle reprend ainsi les termes de la Décision-cadre de l'UE relative à la lutte contre le terrorisme de 2002 22. En outre, la directive inclut une série de modifications dans le domaine préventif, et notamment l'identification de la clientèle et l'obligation de vigilance ${ }^{23}$ pour les institutions financières.

16. Pour une analyse du régime européen de lutte contre le blanchiment d'argent, voir Mitsilegas V., EU Money Laundering Counter-measures, Kluwer Law International, 2003.

17. Troisième directive relative à la prévention de l'utilisation du système financier aux fins $\mathrm{du}$ blanchiment de capitaux et du financement du terrorisme. Directive 2005/60/EC du Parlement et du Conseil du 26 octobre 2005, JO L309, 25 novembre 2005.

18. Considérant 1.

19. Considérant 5.

20. Considérant 9.

21 . Article 3(5)(f). 
L'introduction d'une approche en termes de risque dans le cadre législatif de l'UE est particulièrement importante dans le contexte de l'application des standards du GAFI. Les institutions financières, mais aussi les autres formes d'institutions couvertes par la Directive, sont désormais tenues d'appliquer le principe d'obligation de vigilance en fonction du risque associé au type de client, de relation d'affaires, de produit ou de transaction concerné ${ }^{24}$. Sont également à noter la plus grande priorité désormais accordée aux déclarations de transactions suspectes avec la participation accrue des Cellules de renseignement financier $(\mathrm{CRF})$, dont les modalités des déclarations sont désormais simplifiées afin de garantir la rapidité de leur réception ${ }^{25}$, ainsi que l'accroissement du recours à la « comitologie 26 ", à laquelle est notamment attribuée la clarification des aspects techniques des définitions de concepts clés, tels que "bénéficiaire effectif », "personnes politiquement exposées ", « relation d'affaires », et « société bancaire écran » 27.

La troisième directive relative au blanchiment d'argent a ainsi été constamment justifiée par la nécessité de conformer le cadre législatif de l'UE à l'actualité des règles du GAFI. Un certain nombre de défis sont à noter quant à l'introduction de cette troisième directive relative à la lutte contre le blanchiment d'argent. On peut d'abord mentionner l'inclusion du financement du terrorisme dans les logiques de régulation anti-blanchiment. En effet, celui-ci ne nécessite pas nécessairement de blanchir de l'argent 28 car il est possible d'avoir recours à des fonds parfaitement légaux et légitimes ${ }^{29}$. De plus, les activités terroristes requièrent bien souvent de petites sommes ${ }^{30}$ et peuvent donner lieu à des transactions en dehors du système financier ${ }^{31}$, et comme l'ont souligné de nombreuses études, le régime actuel censé réguler les flux illicites et renforcer les pratiques dans ce domaine, est inefficace et contreproductif ${ }^{32}$. Il apparait, en effet, que l'approche utilisée dans la lutte contre le finan-

22. Article 1(4). Le «financement du terrorisme » est ainsi défini : «le fait, par quelque moyen que ce soit, directement ou indirectement, de fournir ou de réunir des fonds dans l'intention de les voir utilisés ou en sachant qu'ils seront utilisés, en tout ou en partie, en vue de commettre l'une quelconque des infractions visées aux articles 1er à 4 de la décision-cadre 2002/475/JAI du Conseil du 13 juin 2002 relative à la lutte contre le terrorisme ».

23. Le chapitre II de la directive est désormais intitulé « Obligation de vigilance à l'égard de la clientèle » et comprend pas moins de 15 articles - Articles 6-19 de la directive.

24. Articles 8(2), 11(2), 13(1).

25. A ce titre, il est intéressant de noter le chevauchement avec la décision sur les CRF de 2000 (Décision 2000/642/JAI, JO L271, 24 octobre 2000).

26. Articles 40 et 41 .

27. Chapitre VI, Article 40(1)(a).

28. Mitsilegas V., "Countering the Chameleon Threat of Dirty Money. 'Hard' and 'Soft' Law in the Emergence of a Global Regime Against Money Laundering and Terrorist Finance", op. cit. ; Van Duyne P., "Greasing the Organisation of Crime-Markets in Europe", in Van Duyne P., Von Lample K., Newell J. (eds.), Criminal Finances and Organised Crime in Europe, WLP, 2003, pp. 1-17.

29. Mitsilegas V., "From National to Global, from Empirical to Legal: The Ambivalent Concept of Transnational Organized Crime", in Beare M., op. cit., 2003.

30. Levi M., "Criminal Asset-Striping. Confiscating the Proceeds of Crime in England and Wales", in Edwards A., Gill P., op. cit., 2003. 
cement du terrorisme produit l'inverse des effets recherchés, y compris dans le coût élevé de remise de fonds, une marge de manœuvre moins importante pour les services de remise et de transfert de fonds, une criminalisation inutile de certains secteurs économiques et de certaines minorités ethniques, une réduction de la transparence et de la traçabilité des transactions, une relation de confiance endommagée entre les communautés ethniques et les autorités officielles ${ }^{33}$. La consolidation de l'extension aux professions juridiques des obligations dans la lutte contre le blanchiment constitue un autre élément de préoccupation. Cet aspect peut avoir des répercussions sur les droits fondamentaux, et notamment le droit au principe d'équité dans les Etats membres ${ }^{34}$. Le rôle délicat assigné aux banquiers est désormais étendu aux avocats, aux comptables, aux agents immobiliers. Pour les avocats, cela n'a pas seulement des conséquences sur le secret professionnel, cela peut également menacer le droit de tout accusé à un procès équitable ${ }^{35}$. En outre, on demande aux Etats membres de créer des CRF dont les tâches sont précisées et ces cellules se voient accorder un pouvoir d'accès maximal aux bases de données nationales. Pourtant, aucune mesure de protection de ces données n'accompagne cet accès maximal ${ }^{36}$. Le recours croissant à la comitologie introduite dans cette troisième directive afin de définir et d'étendre des concepts clés dans la lutte contre le blanchiment d'argent est en outre problématique. Or, ces concepts sont centraux dans la délimitation des obligations telles que présentées dans la directive, qui reprend ainsi les règles du GAFI. Leurs définitions peuvent avoir des conséquences importantes sur la responsabilité des institutions et des personnes impliquées, mais aussi sur les droits fondamentaux de ces dernières (et notamment ce qu'est une " personne politiquement exposée ») ${ }^{37}$. De plus, ce recours à la comitologie limite considérablement le droit de regard sur l'élaboration et la définition des concepts pourtant centraux. Enfin, avec l'introduction de cette troisième directive, des inquiétudes ont été soulevées par ce qu'on peut appeler une « lassitude d'application », cette directive intervenant effectivement très peu de temps après les délais d'application de la seconde. De manière plus générale, le rôle de leadership assumé par le GAFI et son influence sur les développements des outils de l'UE, doivent être

31. Naylor, R.T., Satanic Purses: Money, Myth, and Misinformation in the War on Terror, op. cit.

32 . Bierstecker T., Eckert S. (eds.), Countering the Financing of Terrorism, NY, Routledge, 2007; Wade I., The Price of Fear: The Truth behind the War on Terror, London, Tauris \& Co, 2007.

33. Passas N., "Fighting Terror with Error: The Counter-Productive Regulation of Informal Value Transfers", Crime, Law E Social Change, n45, 2006, pp. 315-336.

34 . En effet, le Parlement européen a soulevé avec force ces préoccupations lors des négociations de la 2 de Directive sur le blanchiment d'argent. Voir aussi le jugement de la CEJ, Affaire 305/05, 26 juin 2007.

35 . Mitsilegas V., "From National to Global, from Empirical to Legal: The Ambivalent Concept of Transnational Organized Crime", op. cit.

36. Article 21. Selon le paragraphe 3, les Etats membres doivent s'assurer que les CRF ont accès, directement ou indirectement, et ce de manière régulière, aux informations qu'elles jugent utiles dans leurs tâches, et notamment celles d'ordre financier, administratif et policier, qu'elles jugent utiles dans leurs tâches.

37. Dans ce contexte, une directive de la Commission a été adoptée en 2006. Voir Directive 2006/70/CE, JO L214, 4 Août 2006, p. 29. 
remis en cause. Le GAFI fait constamment l'objet de critiques, aussi bien pour les orientations qu'il promeut au niveau international que pour son absence de légitimité ${ }^{38}$. En effet, il s'agit d'une instance ad hoc, dont les membres sont essentiellement ceux de pays riches, et n'a donc pas la légitimité d'une organisation internationale.

\section{La corruption}

Dans le domaine de la corruption, l'UE a été très active, avec l'adoption de standards destinés aux pays candidats à l'entrée dans l'UE. Elle a également participé à l'élaboration d'initiatives internationales dans ce domaine. Toutefois, force est de constater que l'action législative interne de l'UE contre la corruption n'a pas été aussi intense, tout du moins en comparaison avec les actions similaires dans le domaine du crime organisé et du blanchiment d'argent. Les instruments législatifs adoptés dans le cadre de l'UE contre la corruption datent du système de Maastricht - notamment la convention visant à lutter contre la corruption impliquant des fonctionnaires 39 et le premier protocole à la Convention relative à la protection des intérêts financiers des Communautés européennes ${ }^{40}$. Ces deux instruments tentent de définir et de criminaliser la corruption de fonctionnaires, et le protocole confirme la prise en compte du lien entre corruption et fraude au budget de l'Union. La corruption dans le secteur privé est traitée dans une décision-cadre datant de 2003 41, qui criminalise la corruption dans ce secteur. Cependant, les Etats membres trouvent cette décision difficile à mettre en œuvre ${ }^{42}$.

Il apparaît clairement que la stratégie de l'UE suit la tendance globale qui va vers une maximisation de la lutte contre le blanchiment d'argent et le financement du terrorisme, tandis que la mobilisation se fait plus discrète dans le domaine, précisément, de la corruption. L'adoption d'un réseau de points de contact contre la corruption, de même que les efforts entrepris par la Commission européenne afin de comprendre les liens entre criminalité organisée et corruption (comme en témoigne la préparation d'un document de travail de février 2008 portant sur l'examen des liens entre la criminalité organisée et la corruption ), constituent certainement des avancées supplémentaires dans la stratégie de l'UE contre la corruption ${ }^{43}$. Toutefois, comme indiqué précédemment, alors que l'UE a été extrêmement active dans l'adoption de

38. Voir sur ce point, Scherrer A., «La circulation des normes dans le domaine du blanchiment d'argent : le rôle du G7/8 dans la création d'un régime global ", Cultures E Conflits, 2006, $\mathrm{n}^{\circ} 62$, pp. 129-148.

39. JO C195, 25 juin 1997.

40. JO C313, 23 octobre 1996.

41. JO L192, 31 juillet 2003.

42. Voir COM (2007) 328 final. Le document indique notamment : « En ce qui concerne l'article 2 de la décision-cadre, la Commission note que la transposition de cette "article clé" définissant les actes de corruption active et passive constitutifs d'une infraction pénale est une tâche ardue pour la plupart des Etats». 
règles anti-corruption à l'égard des Etats candidats à l'adhésion, la législation interne de l'UE n'a pas été aussi intense. L'approche législative de l'UE contre la corruption, à la fois dans le secteur public et le secteur privé, est par conséquent largement incomplète. Si la Convention de Mérida des Nations unies inclut des mesures contre la corruption dans le secteur privé, il convient de noter qu'elle n'opère qu'une timide avancée, et que la communauté internationale dans son ensemble demeure discrète sur cette question, surtout en comparaison avec l'unanimité assourdissante qui se fait autour du financement du terrorisme et du blanchiment d'argent. Le silence de la communauté internationale sur le crime d'entreprise est également surprenant. De récents exemples (comme les affaires Enron et WorldCom) ont montré que la fraude et la corruption pouvaient toucher de nombreuses industries avec, pour conséquence, des pertes financières massives et de nombreuses victimes, et des centaines de mises en examen ${ }^{44}$. Comme rappellent certains chercheurs à ce propos, il y a peu d'obstacles juridiques qui pourraient empêcher d'assimiler la criminalité en col blanc à du crime organisé : les définitions de l'UE et celles de la convention de Palerme de 2000 s'appliquent en effet à un large éventail d'acteurs et d'activités, le seuil de deux ou trois participants étant facilement franchissable. Cependant, les représentations sociales et policières qui accompagnent le concept vague de « criminalité organisée » demeurent totalement prisonnières de l'image des organisations de type mafieuse, dont les acteurs seraient en marge de tout système économique et social ${ }^{45}$.

\section{La coopération opérationnelle au sein de l'UE}

Cette seconde partie a pour objet la description des principaux dispositifs européens d'entraide et de coopération mis en œuvre pour lutter contre la criminalité organisée transnationale. Après une présentation des premiers instruments d'entraide judiciaire créés durant les années 1990 (magistrats de liaison, réseau judicaire européen), les deux agences européennes en charge de cette lutte (Europol et Eurojust) seront examinées. La compréhension des structures et des pratiques quotidiennes de ces agences conduit à poser la question de leur contrôle aussi bien au niveau de leur champ de compétence que de celui de la disponibilité des informations dont elles disposent.

43. La mise en place d'un réseau de points de contact contre la corruption (suite aux recommandations du Rapport Pirker) constitue à ce titre un élément significatif du Conseil JAI de l'UE d'octobre 2008.

44. Tillman R., "Making the Rules and Breaking the Rules: The Political Origins of Corporate Corruption in the New Economy”, Crime, Law E Social Change, n51, 2009, pp. 73-86.

45 . Levi M., "White-collar, Organised and Cyber Crimes in the Media: Some Contrasts and Similarities”, Crime, Law E Social Change, n49, 2008, pp. 365-377.

46. Action commune 96/277/JAI, du 22 avril 1996, concernant un cadre d'échange de magistrats de liaison visant à l'amélioration de la coopération judiciaire entre les Etats membres de l’Union européenne, JO L105 du 27 avril 1996. 
Les premiers échanges de magistrats de liaison ont eu lieu entre la France et l'Italie suite à l'attentat du 23 mai 1992 contre le juge Giovanni Falcone, figure charismatique de la lutte anti-mafia. Nommés par les chancelleries nationales, les magistrats de liaison travaillent auprès des autorités judiciaires centrales de l'Etat d'accueil. Cette « bonne pratique européenne » depuis 1996 inclut comme principales missions de contribuer à l'amélioration du traitement des dossiers d'entraide dans le domaine de l'assistance répressive, des procédures d'extradition et des commissions rogatoires internationales, ainsi que de promouvoir les échanges diplomatiques et pratiques entre les Etats ${ }^{46}$. Pour pouvoir remplir ces différents objectifs, le magistrat de liaison n'a pas de pouvoir particulier. Il occupe un poste de magistrat à l'étranger et perd, dès lors, son pouvoir de juge ou de procureur. La plupart des magistrats de liaison rencontrés expliquent que leur agenda de travail est ainsi totalement dépendant des demandes qu'effectuent les magistrats nationaux. Tous les pays membres de l'UE ne bénéficient pas de ce type de dispositifs. La France, avec treize magistrats de liaison dont six en Europe, constitue le pays européen le plus actif dans l'utilisation de ce dispositif.

Le réseau judiciaire européen (RJE) est, quant à lui, pour la première fois officiellement évoqué dans le Programme d'action contre la « criminalité organisée ", approuvé par le Conseil européen le 17 juin 1997 à Amsterdam. Le 29 juin 1998, le Conseil européen adopte une action commune portant création du RJE ${ }^{47}$. Ce dispositif d'entraide s'inscrit dans une approche similaire à celle du système des magistrats de liaison, puisque ses missions sont de faciliter les demandes judiciaires d'entraide relatives aux « formes graves de criminalité organisée ", de coordonner les demandes d'enquête, et de fournir les informations nécessaires aux magistrats de l'UE sur les systèmes juridiques, les textes en vigueur et les règles procédurales. Si, de par ces attributs, la correspondance avec le système des magistrats de liaison apparaît évidente, la principale innovation de ce dispositif repose sur sa composition et son architecture territoriale. Le RJE se compose d'un ensemble de points de contact, quatre cents sur l'ensemble du territoire européen ${ }^{48}$, au sein des différents échelons judiciaires de chaque Etat et au niveau des autorités judiciaires centrales en charge de la coopération internationale. Très récemment, une décision-cadre, adoptée le 16 décembre 2008, a renforcé les capacités du RJE dans ses domaines de compétence ${ }^{49}$.

47. Pour un accès à l'ensemble des informations relatives au RJE, voir le site : http://www.ejncrimjust.europa.eu/about-ejn.aspx

48. Chiffres fournis sur le site du RJE : http://www.ejn-crimjust.europa.eu/about-ejn.aspx

49. Décision-cadre relative au réseau judiciaire européen, 008/976/JAI, JO 348/130, 24 décembre 2008. 
Face à une pluralité d'organisations judiciaires et à la volonté des chancelleries nationales de conserver une indépendance totale sur leur territoire, les dispositifs d'entraide que sont les magistrats de liaison et le RJE se structurent de façon hétérogène en fonction des pays. Ainsi, la composition et le domaine de compétence des membres du RJE varient fortement selon les Etats - allant d'un point de contact à Malte à cinquante six en Italie ${ }^{50}$. Cette diversité se retrouve également au niveau des affaires traitées même si la plupart des pays ne disposent pas de données chiffrées sur le nombre d'interventions effectuées par leurs points de contacts. Entre 1998 et 2004, le point de contact de l'autorité centrale à Londres a traité en moyenne une demande du RJE par semaine alors que le point de contact du ministère de la Justice des Pays-Bas est saisi quotidiennement. Les Pays-Bas constituent le pays le plus sollicité au niveau de l'entraide, de vingt à trente fois plus que les autres Etats de l'UE. Selon des estimations pour l'année 2004, les Pays-Bas ont reçu six cents demandes d'entraide judiciaire et six cents demandes d'entraide policière pour trois cents requêtes transmises ${ }^{51}$.

Pour autant, et selon les témoignages recueillis auprès des différents représentants, les éléments caractérisant l'agenda et le travail pénal des magistrats de liaison et des points de contact du RJE tendent fortement à converger. Cette convergence se situe tout d'abord dans la manière dont ces praticiens définissent les principaux problèmes auxquels ils sont confrontés. Pour l'ensemble de ces acteurs, la principale limite réside dans la différence de législation notamment dans les cas d'opérations transfrontalières en matière d'écoutes ou de saisies. A ces difficultés d'ordre juridique s'ajoutent également des problèmes plus objectifs d'ordre technique concernant la viabilité du réseau de communication du RJE, son niveau de sécurité et ses interactions avec les autres réseaux de coopération judiciaire. Enfin, dans la pratique, il semble qu'en raison des missions similaires dévolues aux magistrats de liaison et aux membres du RJE, ainsi que de la logique bilatérale et horizontale de ces deux instruments, on assiste à une superposition de compétences qui ne permet pas une grande lisibilité, en particulier pour les magistrats nationaux peu rompus aux affaires de coopération européenne. Dans un tel contexte, les contacts se font le plus souvent de manière informelle par courrier électronique ou par téléphone dans le cadre de procédures précises. Les relations personnelles jouent dès lors un rôle central, inscrivant ces dispositifs de coopération dans des pratiques judiciaires ponctuelles, hétéroclites et essentiellement interpersonnelles assez éloignées de la vision harmonisée et stable à l'origine de leur création.

50. Chiffres fournis dans le rapport d'activité du réseau judiciaire européen (RJE), 1998-2001.

51. Ces chiffres ont été donnés par plusieurs magistrats rencontrés. Voir Mégie A., Eurojust et le mandat d'arrêt européen. L'européanisation du pouvoir judiciaire : genèse, luttes et enjeux de la construction de deux dispositifs de coopération pénale, thèse de doctorat sous la direction de Renaud Dehousse, Science-Po Paris, décembre 2007, 400 p.

52 . Bigo D., Polices en réseaux, Paris, Presses de Sciences Po, 1996.

53. Données disponibles dans le rapport annuel 2007. 
Compromis entre deux orientations politiques 52 , la Convention Europol de 1995 définit l'unité de coopération policière comme une structure duale, avec d'une part un service dont la mission est d'analyser et de produire des bases de données (service composé de personnes engagées directement par Europol) et d'autre part, un service d'officiers de liaison en charge de faciliter les coopérations bilatérales et/ou multilatérales entre les Etats membres. Depuis la création de cette unité, on observe une croissance exponentielle du nombre d'analystes, alors que les effectifs des bureaux de liaison connaissent une augmentation plus relative si l'on prend en compte l'élargissement en 2004 à 25 Etats membres. En 1999, Europol est composé de 210 personnes dont 40 officiers de liaison et s'appuie sur un budget de 19 millions d'euros. En 2007, l'unité comprend 114 officiers de liaison pour 421 membres du personnel d'Europol. En 2007, le budget est chiffré à 58940367 euros 53 . Concernant ses compétences, Europol connaît également de profonds changements du fait de l'adoption en 2000, 2002 et 2003 de protocoles modifiant la Convention de 1995 qui l'avait créé. Ces protocoles ont mis en moyenne plus de cinq ans pour être ratifiés par l'ensemble des pays membres. Le protocole de 2002 offre, par exemple, la possibilité à l'unité de demander à un Etat d'ouvrir une enquête sous réserve d'informations suffisantes et de participer à des équipes communes d'enquête, regroupant durant une même affaire des représentants de différentes forces de police européennes. En janvier 2006, sous l'impulsion de la présidence autrichienne, un débat sur l'avenir d'Europol s'est ouvert. Ce débat s'est notamment orienté vers une optimisation de son fonctionnement via la mise en place d'une corporate governance, et le développement de ses capacités opérationnelles. Enfin, aux termes de discussions diplomatiques intenses entre les Etats membres, il est prévu qu'à partir du 1 er janvier 2010 Europol devienne une agence de l'Union européenne ${ }^{54}$. Cette montée en puissance de l'unité policière s'accompagne d'un certain nombre de critiques concernant, notamment, l'importance donnée à l'analyse au détriment de la coopération pratique et la question de l'« interopérabilité » des bases de données telles que le Système d'Information Schengen (SISII), les bases de données Europol et le Système européen d'information sur les visas (Visa Information System - VIS) ${ }^{55}$. Dans ce contexte, le contrôle de ces supports techniques ainsi que la conformité juridique et constitutionnelle des accords et cadres institutionnels qui les sous-tendent, notamment vis-à-vis du respect des libertés publiques ${ }^{56}$, constitue l'enjeu principal du développement de l'unité policière ${ }^{57}$.

54. Mercredi 23 avril 2008, par la Commission européenne, source Europa. Disponible à l'adresse : http://www.libertysecurity.org/article2012.html?var_recherche=Europol

55 . Mitsilegas, V., "What are the main obstacles to police cooperation in the EU?", Briefing Note for the LIBE Committee, January 2006.

56. Brouwer E., "The Other Side of Moon. The Schengen Information System and Human Rights: A Task for National Courts”, CEPS Working Document, n²88, April 2008. 
Constituant une agence dotée de la personnalité juridique, la principale caractéristique de l'unité de coopération judiciaire Eurojust réside dans sa dimension intergouvernementale. Cette architecture institutionnelle a pour conséquence de faire des procureurs siégeant à Eurojust des «membres nationaux », ancrés dans le dispositif judiciaire de leur pays d'origine. Dans cette logique, l'organigramme de l'office se structure autour du collège qui, regroupant l'ensemble des représentants nationaux, constitue l'enceinte décisionnelle principale. Les services administratifs connaissent une évolution croissante depuis 2002, à mettre en relation avec celle du budget général de l'unité. Le personnel administratif est ainsi passé de 6 personnes en 2002 à 113 salariés en 2007. Parallèlement, le budget de l'unité a également connu une forte croissance, passant de 6,2 millions d'euros en 2002 (budget exécuté), à 12,1 millions d'euros en 2005 (budget exécuté) et à 18,4 millions d'euros en 2007, soit une augmentation de $25 \%$ par rapport à l'année civile 2006. On constate toujours à l'heure actuelle une forte différence dans la composition numérique des bureaux nationaux. Cette asymétrie dans les ressources financières et humaines de chaque représentation nationale ${ }^{58}$ se prolonge sur le terrain des compétences judiciaires. La grande majorité des membres nationaux sont les seuls représentants de leur Etat au sein d'Eurojust. En 2005, huit pays avaient nommé en plus de leurs représentants, un adjoint : l'Allemagne, la France, l'Italie, les Pays-Bas, le Portugal, la Finlande, la Suède et le Royaume-Uni 59. En 2007, certains pays ont augmenté le nombre de leurs représentants, notamment la France (5 membres), l'Italie (4 membres) ou encore la République Tchèque (2 membres).

Les Etats n'ont pas donné de pouvoirs particuliers à leurs représentants ${ }^{60}$. Dans la totalité des cas, les membres nationaux n'ont pas de contrôle direct sur les poursuites et enquêtes des autorités judiciaires nationales. En 2007, Eurojust a enregistré plus de 1085 affaires, ce qui représente une augmentation de $40 \%$ par rapport à 2006. Comme pour 2006, le « trafic de stupéfiants » et « l'atteinte au patrimoine ou aux biens publics y compris la fraude » constituent le pourcentage le plus élevé. Le nombre de dossiers concernant le «trafic d'êtres humains » et le «blanchiment d'argent » a de son côté nettement augmenté. Si Eurojust a pour principale mission de favoriser la coopération multilatérale entre les autorités nationales en charge de lutter contre les «formes de criminalité grave », les dossiers bilatéraux continuent de représen-

57. Voir les rapports présents sur le site du Contrôleur européen de la protection des données ; voir également le second rapport d'activité de l'autorité de contrôle commune d'Europol. On pourra aussi consulter Bruggeman W., "What are the options for improving democratic control of Europol and for providing it with adequate operational capabilities?", Studia Diplomatica, vol.59, n¹, 2006, pp. 163-181.

58. Rapport annuel d'Eurojust pour l'année 2007, pp. 72-82.

59. Rapport annuel d'Eurojust pour l'année 2005, p. 113.

60. Rapport annuel d'Eurojust pour l'année 2007, p. 16. 
ter une part écrasante de l'activité (813 dossiers en 2007), par rapport aux actions multilatérales (272 dossiers pour l'année 2007) 61. Enfin, à travers l'adoption par le Conseil JLS, le 24 février 2005, de règles de procédure concernant le traitement et la protection des données à caractère personnel, Eurojust a mis en place un système automatisé de gestion de dossiers (Case Management system - CMS). Le développement de ce dernier a pour finalité l'échange sécurisé d'informations judiciaires entre les membres d'Eurojust et les autorités nationales judiciaires. Dans le cadre du projet E-POC (European Pool against Organised Crime) III, le système de traitement des dossiers a été développé depuis l'année 2006 afin d'apporter des améliorations dans l'échange d'informations entre différentes installations E-POC, présentes au sein des Etats membres. En décembre 2007, la connexion d'Eurojust au système informatique Schengen a été réalisée permettant aux membres nationaux de l'unité d'accéder à des extraits d'information contenus dans le SIS.

\section{Europol et Eurojust dans la nouvelle stratégie de l'UE}

Le devenir d'Europol et Eurojust est central dans la reformulation de la stratégie de l’UE contre la criminalité organisée. Plus particulièrement, la nécessité de l'autonomie et du renforcement de ces deux organes a été constamment soulignée, notamment par le Rapport Newton Dunn ${ }^{62}$ et lors du Conseil JAI d'Octobre 2008. La question du mandat et du champ d'action d'Europol et d'Eurojust est centrale dans les efforts d'amélioration actuels de la coopération policière et judiciaire au sein de l'UE. Cependant, et en complément des remarques précédentes sur la nécessité de parvenir à des définitions rigoureuses afin de clarifier les mandats d'Europol et d'Eurojust, peu d'attention a été portée aux problèmes existants et persistants que rencontrent ces deux agences.

Tout d'abord, il convient d'évoquer les relations entre l'unité Eurojust et l'Office de lutte anti-fraude (OLAF) du fait de la forte porosité qui existe entre leurs domaines de compétence en particulier en matière de lutte contre la criminalité organisée. Le fait que ces deux unités se soient construites sur des principes institutionnels antagonistes, dans une logique de compétition entre les promoteurs d'une approche intergouvernementale et les défenseurs d'une démarche intégrationniste de la coopération judiciaire, a des conséquences sur la façon dont ces deux organes se positionnent l'un par rapport à

61. Il faut cependant noter qu'une nouvelle décision concernant l'avenir d'Eurojust est actuellement en cours de préparation (voir la dernière version de cette Décision en date : Document du Conseil 5447/09, 20 janvier 2009). L'article 9 de cette version donne notamment plus de détails sur les pouvoirs des représentants des Etats membres.

62. En mai 2007, la Commission des libertés civiles, de la justice et des affaires intérieures du Parlement Européen a soumis des recommandations au Conseil sur l'élaboration d'un concept stratégique portant sur la lutte contre la criminalité organisée connues sous le nom de « Bill Newton Dunn Report». Voir : Lutte contre la criminalité organisée : élaboration d'un concept stratégique, référence INI/2006/2094. 
l'autre 63. De par l'architecture européenne, Eurojust traite des affaires concernant le troisième pilier de l'UE, alors que l'OLAF n'est compétent que pour les affaires de détournement des fonds communautaires, c'est-à-dire du premier pilier. Néanmoins, cette distinction institutionnelle n'est pas aussi claire sur le terrain. Au-delà des dispositions juridiques et politiques prônant une étroite collaboration, Eurojust et l'OLAF semblent avoir des difficultés à coopérer et restent attachés à leur autonomie. D’ailleurs, le mémorandum, signé entre les deux organes, laisse une grande marge de manœuvre à chaque institution concernant le partage de l'information et la collaboration pratique. Dans le jeu institutionnel européen, Eurojust et l'OLAF cherchent essentiellement à s'imposer afin d'asseoir leur légitimité en tant qu'acteur central de la coopération judiciaire. Cette compétition se traduit en 2000 , juste après la création officielle d'Eurojust, par la mise en place d'une unité de magistrats au sein de l'instance communautaire. En 2007, Eurojust n'a été sollicité que quatre fois par l'OLAF et une seule réunion de coordination a été organisée ${ }^{64}$.

En outre, les relations problématiques entre les dispositifs européens et les services nationaux (services répressifs et judiciaires) ont un impact direct sur le volume d'affaires traitées dans le cadre de la coopération européenne. Cette dimension se trouve au cœur du projet de «formation du personnel de justice » inscrit lors du dernier Conseil JAI, ayant pour objectif de renforcer les liens de confiance et d'échange entre les représentants des unités européennes et les magistrats et policiers nationaux ${ }^{65}$. Malgré une progression constante des affaires traitées depuis la création d'Europol et d'Eurojust, le constat semble dominé par de fortes incertitudes dues à des contraintes organisationnelles et à un manque d'échanges entre le niveau européen et national. De nos entretiens 66 avec des juges français, belges, néerlandais, italiens et des représentants des services de police exerçant leur pouvoir à l'échelle nationale, il résulte que la majorité d'entre eux se considèrent « éloignés » des problématiques européennes, notamment en raison de la nature des infractions qu'ils traitent (qui n'ont pas forcément une dimension européenne), mais aussi en raison de leur absence de connaissance des dynamiques européennes, tant en termes juridiques que linguistiques. Ces enjeux de connaissances intéressent l'ensemble du domaine de la coopération européenne en matière de sécurité. Pour les agents de l'échelon national, le niveau européen est souvent considéré comme complexe, lointain et demandant du temps que la résolution des infractions ne permet pas toujours. Enfin, les pratiques et usages judiciaires peuvent aussi expliquer certains refus. Le partage d'une instruction avec un autre magistrat ou d'autres policiers, de surcroît étrangers, est souvent assimilé à une perte de pouvoir et d'autorité sur la conduite de la procédure. Face à ces difficultés, Eurojust et Europol focalisent une part importante de leur travail en faveur

63. Mégie A. (dir.), « Arrêter et juger en Europe : genèse, luttes et enjeux de la coopération pénale ", Cultures E Conflits, nº2, printemps 2006.

64. Rapport annuel d'Eurojust pour l'année 2007, p. 23.

65. Conseil JAI, 24 octobre 2008, p. 22. 
d'un renforcement des liens avec les autorités nationales. Participant à la stratégie de légitimation, cette démarche représente une part essentielle de l'activité des membres de l'unité Eurojust et des officiers de liaison d'Europol. L'importance des ressources individuelles des membres nationaux prend dans ce contexte une valeur décisive. Dans cette logique, l'accumulation d'une longue expérience judicaire et policière ainsi que la possibilité de s'appuyer sur des réseaux personnels multiples sont des voies de recours privilégiées.

Dans le cas d'Eurojust et d'Europol, si les blocages se situent essentiellement du côté des autorités nationales, il est essentiel de noter que les procureurs ou les officiers de liaison ne s'inscrivent que très peu dans une logique d'autonomisation vis-à-vis de l'échelon national. Dans le cas d'Eurojust, l'utilisation extrêmement rare des articles 6 et 7 de la décision-cadre de 2002, permettant d'émettre une requête officielle auprès des autorités nationales non disposées à soumettre un dossier, en apporte la preuve. Entre 2002 et 2005, seulement deux saisines de ce type ont été réalisées. En réalité, il semble que, malgré la dimension non contraignante de ce type de demande, les membres nationaux hésitent à utiliser une telle procédure afin de ne pas créer de conflits avec les autorités nationales de leur propre pays, qui peuvent considérer ce recours comme une atteinte à leur indépendance. Pour les membres d'Eurojust, l'emploi constant de telles prérogatives constitue principalement un obstacle à leur travail de sensibilisation en direction des confrères du niveau national. La voie informelle et personnelle est, dans ces conditions, très largement privilégiée afin d'établir des relations de «confiance ». Dans ce souci de légitimité et de reconnaissance vis-à-vis des autorités nationales, Eurojust a par exemple décidé depuis 2007 de financer les dépenses des participants nationaux aux réunions de coordination dans ses locaux ou ailleurs en Europe.

Enfin, les rapports de coopération entre Europol et Eurojust restent principalement marqués par des logiques de défiance, voire une certaine rivalité rendant la collaboration entre ces deux instances encore relative et instable. L'absence d'une coopération étroite entre ces unités s'inscrit dans des dynamiques de compétition qui doivent se comprendre en raison d'une superposition de compétences entre ces offices, ainsi que d'une institutionnalisation du champ européen de la sécurité encore en cours ${ }^{67}$. Malgré les dispositions juridiques en faveur d'un renforcement de la collaboration ${ }^{68}$, les relations entre Eurojust et Europol restent distantes. Dans cet environnement, le contrôle de l'information constitue sans aucun doute l'un des enjeux de la compétition qui existe entre les deux unités. Les statistiques relatives aux échanges d'informa-

66. Mégie A., Eurojust et le mandat d'arrêt européen : l'européanisation du pouvoir judiciaire, op. cit.

67. Bigo D., Bonditti P., Mégie A. et al., The Field of the EU Internal Security Agencies, Paris, Centre d'études sur les conflits/Harmattan, 2008.

68. Voir l'Accord entre Eurojust et Europol, 2004. 
tions depuis 2004 entre les deux offices illustrent le peu de collaboration effective. En 2004, aucune demande n'a été émise par Europol auprès d'Eurojust. En 2005, Europol a émis une demande et a reçu une réponse. Dans le sens inverse, l'unité Eurojust a émis huit demandes et a reçu quatre réponses en 2004. Pour l'année 2005, Eurojust a émis 64 demandes et a reçu 52 réponses. Les récents chiffres fournis par les deux unités concernant leur coopération ainsi que les projets conjoints semblent néanmoins marquer les prémices d'une collaboration plus intense. En 2007, un groupe de travail EuropolEurojust sur les fichiers d'analyse (AWF) a été créé afin d'examiner les problématiques juridiques et pratiques de l'assistance d'Eurojust. Le nombre de dossiers traités par Eurojust et impliquant Europol a connu une forte augmentation en 2007, passant de 7 affaires en 2006 à 25 en 2007. Enfin, douze réunions de coordination entre les deux unités ont été organisées pour cette même année 2007 69. Dans cette logique interinstitutionnelle, chaque unité tend à redéfinir de manière extensive son champ de compétence afin de pouvoir légitimer sa place et son rôle dans le champ européen de la sécurité. La manière dont Eurojust a fortement investi le domaine du terrorisme suite aux attentats du 11 septembre illustre ce type de processus 70 .

Dans ce contexte d'absence de clarté des champs d'action des agences de coopération judiciaire et policière au sein de l'UE, les questions du contrôle et de la disponibilité des informations, ainsi que celle de l'interopérabilité des bases de données deviennent centrales ${ }^{71}$. Comme souligné par ailleurs ${ }^{72}$, ces deux aspects de la coopération policière et judiciaire sont problématiques, et ce à plusieurs niveaux : outre les difficultés opérationnelles inhérentes à ce type de gestion des fichiers (travail supplémentaire, lourdeur de la gestion des entrées des données, temps passé à traiter les demandes des autres autorités), et la question du rôle d'Europol dans la mise en place de ces fichiers, le débat porte tout particulièrement sur des raisons touchant à la légitimité même du principe de disponibilité et à ses effets sur les libertés publiques. Ces débats insistent notamment sur la différence qualitative entre les données partagées par les services de renseignements et les services des autorités répressives (polices, douanes, autorités judiciaires) dont l'admissibilité devant un tribunal n'est pas la même, et dont la crédibilité, la véracité dépendent des conditions mêmes dans lesquelles les informations ont été obtenues. Le principe de disponibilité suppose en effet, pour fonctionner, que l'on s'entende sur les catégories concernant les autorités qui auront accès à ces données. Les considérations relatives à la création d'un Système européen d'information sur les

69. Rapport annuel d'Eurojust pour l'année 2007, p. 23.

70. Mégie A., "L'institutionnalisation d'un pouvoir judiciaire européen incertain en quête de légitimité : l'unité de coopération Eurojust ", Politique européenne, n²3, 2007, Paris, l'Harmattan.

71 . Mitsilegas, V., « La coopération policière : quels sont les obstacles majeurs de la coopération policière au sein de l'UE », 2006, note pour le Parlement européen, IP/C/LIBE/FWC/200524. 
casiers judiciaires (ECRIS) ouvrent plus largement encore le débat sur la protection des données et aux principes de disponibilité et d'interopérabilité. Le Conseil JAI d'octobre 2008 s'est en effet accordé sur une approche générale concernant la proposition relative à la création d'un tel système. Cette proposition se situe dans la continuité de la décision-cadre sur l'organisation et le contenu de l'échange d'informations extraites des casiers judiciaires entre les Etats membres, sur laquelle le Conseil s'était accordé en juin 2007. La décision-cadre a pour objectif principal de garantir qu'un Etat membre soit en mesure d'informer les autorités judiciaires d'un autre Etat de l'UE sur le passé pénal de ses ressortissants. La décision ECRIS vise notamment à faire en sorte que les informations soient transmises de manière électronique, et définit les conditions et le format d'échange des données. Les Etats membres seront donc pleinement responsables de la gestion de leurs casiers judiciaires, mais les transferts d'informations seront facilités grâce à un format commun d'échange de données.

À cet égard, comme souligné dans le rapport parlementaire NewtonDunn, la nécessité d'améliorer l'échange de données au sein de l'UE appelle des mesures de protection fiables et viables, en particulier en ce qui concerne la protection des données personnelles. Avant la réunion du Conseil d'octobre 2008, le Contrôleur européen de la protection des données (CEPD), a publié un avis sur la création d'ECRIS ${ }^{73}$, dans lequel ces motifs d'inquiétude étaient également mentionnés, ainsi que le besoin d'améliorer les mécanismes de protection des données au sein de l'UE. Le CEPD, en particulier, a souligné la nécessité d'ajouter des garanties supplémentaires sur la protection des données, pour compenser le manque actuel d'un cadre juridique complet en la matière dans le domaine de la coopération entre polices et autorités judiciaires. En conséquence, il a insisté sur la nécessité d'une réelle coordination du contrôle du système de protection des données, qui implique les autorités des Etats membres et la Commission en tant que fournisseur de l'infrastructure commune de communication. Les modalités de mise en place du système ECRIS doivent ainsi requérir toute l'attention et le suivi nécessaires. Comme le suggérait le Rapport Newton Dunn, l'Agence des droits fondamentaux nouvellement créée pourrait être mobilisée afin de protéger les droits prévus dans la Charte des droits fondamentaux et d'assurer que des garanties de protection soient données à l'extension des échanges de données. Ainsi, si le rapport susmentionné insistait fermement sur la nécessité d'assurer le contrôle parlementaire des actions d'Europol et d'Eurojust, il recommandait fortement

72. Bigo D., Bruggeman W., Burgess P., Mitsilegas V., « Principe de disponibilité des informations ", Policy recommendations, Programme européen Challenge Liberty and Security, 2007. Disponible à l'adresse : http://www.libertysecurity.org/article1377.html

73. «Avis du CEPD sur le système européen d'information sur les casiers judiciaires : nécessité d'une infrastructure solide, d'une information de qualité et d'un contrôle adéquat ", EDPS/08/9, 18/09/2008. Une décision concernant ECRIS a été récemment publiée au Journal Officiel. Voir JO L93, 7 avril 2009, p. 33. 
la nécessité de sauvegardes en particulier en ce qui concerne la protection des données personnelles dans le cadre du troisième pilier. Cependant, la récente décision-cadre relative à la protection des données à caractère personnel traitées dans le cadre de la coopération policière et judiciaire en matière pénale (novembre 2008) n'offre sans doute pas les garanties nécessaires ${ }^{74}$. 